\title{
Préface de la troisième édition
}

Les éditions précédentes du livre de Michel Le Bellac ont rencontré un succès mérité, car elles concilient de façon exceptionnelle clarté et rigueur sans pour autant sacrifier la concision. Donner autant d'information utile dans un nombre de pages raisonnable rend le livre particulièrement agréable à utiliser. La toute dernière édition augmente encore la portée de l'ouvrage, puisqu'elle bénéficie d'additions et de réorganisations qui modifient sensiblement le développement des chapitres. L'intrication et la non-localité quantiques font maintenant l'objet d'un chapitre particulier très riche couvrant, non seulement divers aspects du théorème de Bell, mais également les relations entre décohérence et mesure et l'information quantique. Des exercices corrigés originaux complètent encore l'information, comme par exemple celui qui discute la réalisation de portes quantiques avec des ions. De même, la quantification du champ électromagnétique est maintenant promue au niveau d'un chapitre entier (alors qu'antérieurement, elle était une partie du chapitre sur l'oscillateur harmonique); divers aspects sont traités, comme l'effet d'une lame semi-réfléchissante sur les modes du champ, ou encore l'hamiltonien de Jaynes-Cummings. La théorie de la diffusion est maintenant enrichie d'une partie formelle et opératorielle (équation de Lippmann-Schwinger, matrice T, etc.).

Une addition notable est celle d'un chapitre 19, "Physique quantique relativiste". Une première partie est consacrée à l'analyse de Wigner des propriétés universelles d'un système quantique élémentaire déduites des symétries du groupe de Poincaré. C'est important car cela permet de comprendre l'origine profonde de la masse et du spin en termes de symétries fondamentales. Il est curieux que relativement peu d'ouvrages d'enseignement traitent ce sujet. Cette analyse conduit à l'équation de Dirac, dont les propriétés sont discutées. La quantification de l'équation de Klein-Gordon et de l'équation de Dirac, considérées comme des équations classiques, donne un aperçu utile sur la théorie relativiste des champs. 
À tout ceci s'ajoute un grand nombre d'exercices très riches munis de leurs corrigés, parmi lesquels on peut citer les forces de Van des Waals et l'effet Casimir, la précession de Thomas, le modèle en couches des noyaux, l'étude des expériences de création d'états de Fock pour des photons en cavité, la capture radiative de neutrons par le proton, la hiérarchie BBGKY, les équations pilotes (Lindblad, Fokker-Planck), etc. De nombreuses figures illustrent le texte d'une façon qui en rend l'accès plus agréable au lecteur.

Bref, plus encore dans cette nouvelle version, cet ouvrage est un concentré d'informations et d'idées dans presque tous les domaines qui touchent au quantique, qui servira beaucoup non seulement aux étudiants, mais également aux physiciens des laboratoires comme ouvrage de référence.

Franck Laloë 